\title{
Desempeño financiero de empresas que emiten bonos corporativos
}

DOI del artículo: 10.36631/ECO.2021.25.03

Artículo de reflexión

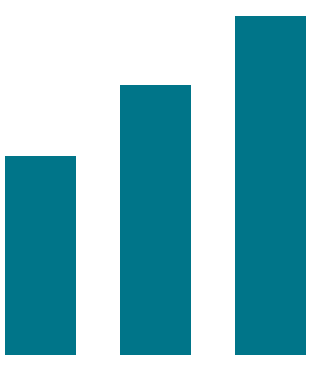

\section{Guillermo Díaz}

Facultad de Ciencias Económicas y Empresariales, Universidad Rafael Landívar

Correo: godiaz@url.edu.gt

Fecha de recepción: 4/6/2021

Fecha de aceptación: 23/7/2021

\section{Resumen}

En el presente artículo se realiza un análisis del desempeño financiero de una muestra de diez empresas guatemaltecas, emisoras de bonos corporativos en el mercado nacional e internacional. Para el análisis se utilizaron los métodos EVA y Dupont. Los resultados indican que la mayoría de empresas generan valor y que el mismo es producto de estrategias basadas en margen y volumen.
Palabras clave: EVA, valor económico agregado, Dupont, desempeño financiero, rentabilidad de capital 


\section{FINANCIAL PERFORMANCE IN FIRMS THAT ISSUE CORPORATE BONDS}

\section{Reflection paper}

\section{Abstract}

This article presents an analysis of the financial performance of a group of ten Guatemalan firms that issue corporate bonds both for the national and international markets. For this analysis, the EVA and Dupont were used. Results indicated that most of these companies generate value as a result of strategies based on margin and volume Keywords: EVA, added economic value, Dupont, financial performance, capital yield 


\section{Introducción}

En el ámbito financiero, las empresas, en general, miden su desempeño con base en el monto de ganancias obtenidas en el año contable o calendario, en comparación con el anterior. También suelen utilizar el indicador de rentabilidad sobre capital, para tal fin. En el primer caso, el énfasis está en obtener un monto mayor cada año y, en el segundo, en obtener una tasa de rentabilidad igual o mayor que la del año previo o la del sector en que se compite. La importancia de la generación de ganancias es tal que en el mercado de capitales se utiliza la relación precio/ganancias para determinar el precio hipotético de la misma (Gitman y Joehnk, 2009).

Si bien la obtención de ganancias y la rentabilidad son indicadores adecuados para medir el desempeño financiero de una empresa, también es importante conocer si las ganancias que se generan reditúan el costo del capital utilizado en su generación, si generan valor y cómo se generan dichas ganancias. En el primer caso, el Valor Económico Agregado (EVA) es útil para conocer si el beneficio económico es mayor que el costo del capital invertido. En el segundo, el método Dupont es útil para desglosar la obtención de la rentabilidad, a través de sus tres componentes: margen, eficiencia (rotación de activos) y apalancamiento (estructura del balance). En la práctica, el método Dupont permite saber si la rentabilidad es resultado de un modelo de negocio de margen, de volumen o de la estructura financiera (Quemada, 2016).

En este artículo se utiliza el método del EVA y el de Dupont para evaluar el desempeño financiero de empresas guatemaltecas emisoras de bonos corporativos en mercados internacionales. Ambos métodos han sido utilizados en estudios o investigaciones similares a la que se presenta en esta ocasión. Entre ellos se puede citar los de Godoy, Acero y Rivero (2011), para el sector de alimentos y bebidas de Colombia; el de Tellez (205), para empresas que cotizan en la bolsa de valores de México; el de Arellanos (2016), para empresas industriales de Perú y el de Valdés, Martínez y Beltrán (2020), para el caso de Cemex en México. Una investigación relevante para la presente es la realizada por Quichimbo (2019), porque utilizó ambos métodos para analizar la rentabilidad de cooperativas de ahorro y crédito de Ecuador.

El análisis de desempeño financiero que se realiza en este artículo utiliza ambos métodos porque se consideran complementarios. El primero, como ya se dijo, indica cuanto valor genera la empresa, mientras que el segundo revela las fuentes de dicho valor o modelo de negocio. 


\section{Método}

El EVA es un método creado por Stern Stewart \& Co y constituye una estimación de la creación o destrucción de valor de la empresa. Mide si las ganancias son suficientes para cubrir el costo del capital empleado en generarlas. El EVA puede ser estimado con base en las siguientes dos ecuaciones (Desai y Ferri, 2006):

\section{$E V A=$ Utilidad operativa neta $-($ Costo de capital X Capital $)$}

$$
E V A=(R O E-\text { costo de capital }) * \text { Capital }
$$

El EVA, como indicador de evaluación del desempeño financiero, es superior a otros, como el de la rentabilidad de capital o el Dupont, porque evalúa la rentabilidad económica y no la rentabilidad contable. La primera se basa en generación de flujos de caja y la segunda en datos contables, que pueden tener un costo asignado o no, mientras que los recursos utilizados en producir los flujos de caja tienen un costo económico de oportunidad. En consecuencia, la creación de valor o rentabilidad económica se asocia a un nivel de riesgo (Bonilla, 2010).

El método Dupont permite evaluar el rendimiento sobre los activos y sobre el capital, dando al inversionista, accionista o gerente la oportunidad de analizar cómo mejorar la rentabilidad. El método combina indicadores financieros del balance general y del estado de resultados, como lo son rendimiento sobre activos y rendimiento sobre capital, utilizando el grado de apalancamiento financiero (García, 2014).

Siguiendo a Granel (2020), el método Dupont permite analizar la rentabilidad de una empresa que usa indicadores tradicionales de desempeño, a través de la siguiente fórmula:

$$
R O E=\frac{\text { Ingreso neto }}{\text { Ventas }} \times \frac{\text { Ventas }}{\text { Activos }} \times \frac{\text { Activos }}{\text { Capital }}
$$

La primera expresión de la anterior ecuación (ingreso neto/ventas) constituye el margen de utilidad y es útil para conocer qué porcentaje de las ventas se convierten en ganancias. El segundo elemento (ventas/activos) es la rotación de activos y es un indicador de eficiencia del uso de los mismos, en el sentido de medir su productividad para generar ventas. El aumento de dicho indicador significa que la empresa es más eficiente en gestionar sus recursos. El tercer indicador del método Dupont es el de apalancamiento e indica la cantidad de recursos externos que utiliza la empresa para financiar sus operaciones; entre mayor sea dicho índice más contribuye a aumentar la rentabilidad sobre capital, ya que este se utiliza menos en el financiamiento de la empresa (Westreicher, 2020). 


\section{Datos}

Las empresas seleccionadas para evaluar su desempeño financiero fueron diez, de las cuales cinco emitieron bonos corporativos en el mercado internacional entre 2010 y 2012 y las otras cinco los emitieron en el mercado nacional entre 2014 y 2015. Al respecto, para el análisis comparativo se asume indiferencia en relación al período de tiempo de la emisión. De las cinco empresas que emitieron bonos en el mercado internacional, dos fueron industriales, siendo estas Cementos Progreso y $C B C$, esta última dedicada a la manufactura de bebidas carbonatadas; dos fueron bancos, Industrial y De los Trabajadores, y una fue de servicios de telefonía, Tigo. De las cinco empresas que emitieron bonos en el mercado nacional, dos fueron financieras, emisoras de tarjetas de crédito: Conticredit y Credomatic; dos agroindustriales, San Diego y Hulatex, y una inmobiliaria, Inversiones Preferentes.

En los cinco casos de las empresas emisoras en mercados internacionales de deuda, el prospecto de la emisión de los bonos se registró en la bolsa de valores de Luxemburgo, de donde se obtuvo acceso a dicho documento que contiene los estados financieros, para calcular los indicadores de Dupont y el EVA. En el cuadro 1 se presentan los datos de las cuentas bancarias utilizadas para el cálculo del Dupont y el EVA. En dicho anexo se puede observar que la empresa más grande, según monto de los activos y ventas, es Banco Industrial, con valores de $\mathrm{Q}_{50}$ ooo y $\mathrm{Q}_{1} 615$ millones, respectivamente, para el año 2010. La segunda empresa más grande según valor de los activos fue banco De los Trabajadores, con Q9 623 millones en 2012, seguido de Tigo, con Q1 241 millones en ese año.

Tabla 1

Valores de cuentas contables para el cálculo del Dupont y el EVA

\begin{tabular}{|c|r|r|r|r|r|}
\hline $\begin{array}{c}\text { Cuenta. Valores } \\
\text { en millones de } \\
\text { quetzales }\end{array}$ & $\begin{array}{c}\text { Cementos } \\
\text { Progreso }\end{array}$ & \multicolumn{1}{c|}{ CBC } & \multicolumn{1}{|c|}{ Tigo } & Bantrab & Industrial \\
\hline & 2012 & \multicolumn{1}{|c|}{2012} & \multicolumn{1}{c|}{2012} & \multicolumn{1}{c|}{2012} & \multicolumn{1}{|c|}{2010} \\
\hline Utilidad & 90.3 & 39.2 & 438.3 & 131.1 & 763.3 \\
\hline Ventas & 507.7 & 1092.6 & 1111.5 & 1542.9 & 1614.9 \\
\hline Activo & 1161.1 & 1006.7 & 1241.0 & 9623.8 & 50064.8 \\
\hline Deuda & 481.1 & 746.8 & 712.8 & 8796.1 & 45686.1 \\
\hline Capital & 680.0 & 260.0 & 528.2 & 827.7 & 4378.7 \\
\hline
\end{tabular}

Fuente: elaboración propia, con base en datos de la bolsa de valores de Luxemburgo. 
En el caso de las cinco emisoras en el mercado nacional, los datos de los estados financieros se obtuvieron de los prospectos publicados en la Bolsa Nacional de Valores. Los datos de las cuentas contables de estas empresas se presentan en el cuadro 2. En este grupo, también las empresas financieras fueron las más grandes según monto de activos y ventas. La mayor fue Credomatic con activos de $\mathrm{Q}_{3} 260$ millones y ventas de $\mathrm{Q}_{1} 367$ millones en 2016.

Tabla 2

Valores de cuentas contables para el cálculo del Dupont y el EVA

\begin{tabular}{|c|c|c|c|c|c|}
\hline $\begin{array}{c}\text { Cuenta. } \\
\text { Valores en } \\
\text { millones de } \\
\text { quetzales }\end{array}$ & San Diego & $\begin{array}{c}\text { GYT } \\
\text { Conticredit }\end{array}$ & Credomatic & $\begin{array}{c}\text { Inversiones } \\
\text { referentes }\end{array}$ & Hulatex \\
\hline Utilidad & 1.535263 & 54.22178 & 446.3886 & 3.281789 & 3.568017 \\
\hline Ventas & 4.011075 & 158.7857 & 1303.024 & 3.084914 & 3.765991 \\
\hline Activo & 75.83135 & 566.6527 & 3458.86 & 41.774717 & 44.327057 \\
\hline Deuda & 0.387635 & 438.7575 & 2381.571 & 0.089092 & 0.135027 \\
\hline Capital & 75.443715 & 127.8952 & 1077.289 & 41.685625 & 44.19203 \\
\hline
\end{tabular}

Fuente: elaboración propia, con base en datos de la Bolsa de Valores Nacional.

Para el cálculo del costo de capital propio, se utilizó la tasa de interés de los bonos de Estados Unidos a 30 años plazo y la tasa de EMBI para Guatemala, como prima de riesgo. Ambas variables referidas a 2012. La primera a ese año registró un valor de $2.8 \%$ en promedio y la segunda, de $3.7 \%$. En consecuencia, el costo del capital propio fue estimado en $6.5 \%$ anual. Para ambas muestras de empresas se utilizó el mismo costo de capital propio, porque no se consideró necesario calcular ajuste por riesgo cambiario, debido a la estabilidad del tipo de cambio del quetzal respecto al dólar. El costo del capital ajeno o deuda fue estimado para cada empresa con base en los gastos de intereses y el monto de endeudamiento. Dicho costo osciló entre 2 $\%$ y $12 \%$. El costo ponderado de capital fue la suma de ambos costos multiplicado cada uno por el respectivo porcentaje de financiamiento. Dicho costo difiere para cada empresa y fluctuó ente $3 \%$ y $10 \%$. 


\section{Resultados}

El cálculo del EVA revela que todas las empresas creaban valor en el año previo y en el de la emisión de los bonos corporativos en el mercado internacional (ver cuadro 3). Las empresas que más EVA crearon en términos absolutos fueron Banco Industrial y Tigo, ambas empresas de servicios, la primera financieros y la segunda de telecomunicaciones. Las empresas que menos EVA generaron en valor absoluto fueron las industriales, CBC y Cementos Progreso.

El EVA, como porcentaje que mide cuánto supera el costo ponderado de capital, fue mayor en la empresa Tigo, con una tasa promedio de casi $70 \%$ en cada uno en los años 2011 y 2012. La empresa CBC fue la que obtuvo el menor porcentaje, de alrededor de $6 \%$ en promedio en dichos años. Lo anterior significa que fue la empresa Tigo la que más EVA creó en relación al capital invertido, es decir, la que mejor remuneró a los accionistas.

Tabla 3

Cálculo del Valor Económico Agregado (EVA)

\begin{tabular}{|c|c|c|c|c|c|}
\hline & $\begin{array}{c}\text { Cementos } \\
\text { Progreso }\end{array}$ & CBC & Tigo & Bantrab & $\begin{array}{c}\text { Banco } \\
\text { Industrial }\end{array}$ \\
\hline Variables & 2012 & 2012 & 2012 & 2012 & 2010 \\
\hline $\begin{array}{c}\text { Costo ponderado } \\
\text { de capital (A) }\end{array}$ & $4.6 \%$ & $3.4 \%$ & $4.1 \%$ & $5.2 \%$ & $2.7 \%$ \\
\hline $\begin{array}{c}\text { Rentabilidad sobre } \\
\text { capital (B) }\end{array}$ & $13.3 \%$ & $15.1 \%$ & $83.0 \%$ & $15.8 \%$ & $17.4 \%$ \\
\hline $\begin{array}{c}\text { Capital (C) } \\
\text { EVA en porcentaje } \\
\text { (D= B-A) }\end{array}$ & $8.7 \%$ & $11.7 \%$ & $78.8 \%$ & $10.7 \%$ & $14.7 \%$ \\
\hline $\begin{array}{c}\text { EVA en millones de } \\
\text { quetzales (D*C) }\end{array}$ & 59.3 & 260.0 & 528.2 & 827.7 & 4.378 .7 \\
\hline
\end{tabular}

Fuente: elaboración propia, con base en datos del cuadro 1.

En el caso de las empresas que emitieron bonos corporativos en el mercado nacional, los resultados, mostrados en el cuadro 4, indican que fueron las empresas financieras las que más EVA crearon. Credomatic fue la que generó más EVA, con un monto promedio de Q350 millones entre los años 2015 y 2016. Ambas empresas fueron también las que obtuvieron mayor EVA como porcentaje del capital. Las empresas Inversiones Preferentes y Hulatex escasamente crearon EVA, mientras que la empresa San Diego destruyó valor para sus accionistas en los años 2015 y 2016. 
Tabla 4

Cálculo del Valor Económico Agregado (EVA)

\begin{tabular}{|c|r|r|r|c|c|}
\hline & San Diego & $\begin{array}{c}\text { GYT } \\
\text { Conticredit }\end{array}$ & Credomatic & $\begin{array}{c}\text { Inversiones } \\
\text { Preferentes }\end{array}$ & Hulatex \\
\hline Variables & 2015 & 2015 & 2015 & 2015 & 2015 \\
\hline $\begin{array}{c}\text { Costo ponderado } \\
\text { de capital (A) }\end{array}$ & $6.6 \%$ & $9.7 \%$ & $4.7 \%$ & $6.5 \%$ & $6.5 \%$ \\
\hline $\begin{array}{c}\text { Rentabilidad sobre } \\
\text { capital (B) }\end{array}$ & $2.0 \%$ & $42.4 \%$ & $41.4 \%$ & $7.9 \%$ & $8.1 \%$ \\
\hline Capital (C) & 75.4 & 127.9 & 1077.3 & 41.7 & 4.2 \\
\hline $\begin{array}{c}\text { EVA en porcentaje } \\
\text { (D=B-A) }\end{array}$ & $-4.6 \%$ & $32.7 \%$ & $36.7 \%$ & $1.4 \%$ & $1.6 \%$ \\
\hline $\begin{array}{c}\text { EVA en monto } \\
\text { (D*C) }\end{array}$ & -3.5 & 41.9 & 395.3 & 0.6 & 0.7 \\
\hline
\end{tabular}

Fuente: elaboración propia, con base en datos del cuadro 2.

Los cálculos del índice Dupont muestran diferencias en la obtención de las ganancias, por consiguiente, del EVA de las empresas. En las emisoras de deuda corporativa internacional se observa, en el cuadro 5, que el modelo de creación de valor y de rentabilidad en Tigo y Banco Industrial se basa en apalancamiento y margen sobre ventas. En el caso del Banco de los Trabajadores también se basa en apalancamiento, por ser banco como el Industrial, pero a diferencia de este, el segundo factor importante en la generación de valor es la rotación de activos. En las empresas industriales, Cementos Progreso y $C B C$, la rentabilidad y creación de valor se fundamenta en rotación de activos y apalancamiento. Tigo es una empresa que también utiliza el apalancamiento para mejorar sus indicadores de rentabilidad y creación de valor. Es por tal razón que es la empresa con mayor rentabilidad y EVA.

Tabla 5

Cálculo del índice Dupont

\begin{tabular}{|c|c|c|c|c|c|}
\hline & $\begin{array}{c}\text { Cementos } \\
\text { Progreso }\end{array}$ & CBC & Tigo & Bantrab & $\begin{array}{c}\text { Banco } \\
\text { Industrial }\end{array}$ \\
\hline Variables & 2012 & 2012 & 2012 & 2012 & 2010 \\
\hline $\begin{array}{c}\text { Utilidad/ } \\
\text { ventas }\end{array}$ & $17.8 \%$ & $3.6 \%$ & $39.4 \%$ & $8.5 \%$ & $47.3 \%$ \\
\hline $\begin{array}{c}\text { Ventas/ } \\
\text { active }\end{array}$ & $43.7 \%$ & $108.5 \%$ & $89.6 \%$ & $16.0 \%$ & $3.2 \%$ \\
\hline $\begin{array}{c}\text { Deuda/ } \\
\text { active }\end{array}$ & $41.4 \%$ & $74.2 \%$ & $57.4 \%$ & $91.4 \%$ & $91.3 \%$ \\
\hline ROA & $7.8 \%$ & $3.9 \%$ & $35.3 \%$ & $1.4 \%$ & $1.5 \%$ \\
\hline ROE & $13.3 \%$ & $15.1 \%$ & $83.0 \%$ & $15.8 \%$ & $17.4 \%$ \\
\hline
\end{tabular}

Fuente: elaboración propia, con base en datos del cuadro 1. 
En las empresas emisoras en el mercado de deuda nacional, el índice Dupont muestra, en el cuadro 6, que las empresas no financieras, San Diego, Inversiones Preferentes y Hulatex, basan su rentabilidad y creación de valor en el margen sobre ventas. Las empresas financieras, Credomatic y GYT Conticredit, lo basan en el apalancamiento, conforme a su naturaleza de negocio, y lo combinan con margen y rotación de activos.

Tabla 6

Cálculo del índice Dupont

\begin{tabular}{|c|c|c|c|c|c|}
\hline & San Diego & $\begin{array}{c}\text { GYT } \\
\text { Conticredit }\end{array}$ & Credomatic & $\begin{array}{c}\text { Inversiones } \\
\text { Preferentes }\end{array}$ & Hulatex \\
\hline Variables & 2015 & 2015 & 2015 & 2015 & 2015 \\
\hline Utilidad/ventas & $38.3 \%$ & $34.1 \%$ & $34.3 \%$ & $106.4 \%$ & $94.7 \%$ \\
\hline Ventas/active & $5.3 \%$ & $28.0 \%$ & $37.7 \%$ & $7.4 \%$ & $8.5 \%$ \\
\hline Deuda/active & $0.5 \%$ & $77.4 \%$ & $68.9 \%$ & $0.2 \%$ & $0.3 \%$ \\
\hline ROA & $2.0 \%$ & $9.6 \%$ & $12.9 \%$ & $7.9 \%$ & $8.0 \%$ \\
\hline ROE & $2.0 \%$ & $42.4 \%$ & $41.4 \%$ & $7.9 \%$ & $8.1 \%$ \\
\hline
\end{tabular}

Fuente: elaboración propia, con base en datos del cuadro 2. 


\section{Discusión}

En un análisis comparativo realizado entre las diez empresas analizadas, los resultados revelan que las empresas emisoras de bonos corporativos en el mercado internacional crearon más EVA y obtuvieron mejor rentabilidad, que las empresas emisoras en el mercado nacional. De hecho, en este último grupo hubo una empresa, San Diego, que destruyó valor y dos, Inversiones Preferentes y Hulatex, apenas crearon.

Comparado con estudios realizados en Colombia (Escobar, 2008; Pabón, Bastos y Mogrovejo, 2015) y Costa Rica (Alfaro, Castillo y Gutiérrez, 2011) en el sector industrial las empresas guatemaltecas analizadas muestran mejor desempeño en términos del EVA respecto a las empresas colombianas, porque la mayoría crearon valor, mientras que las empresas colombianas analizadas destruyeron valor. Con relación a las empresas costarricenses la situación es similar, dado que estas también crearon valor. En el caso de las empresas financieras, también se observa similitud respecto a cooperativas de ahorro y crédito de Ecuador (Quichimbo, 2019), en lo que respecta a obtener un EVA positivo. El mismo también es similar como porcentaje del capital, alrededor de $12 \%$. Lo mismo se observa en el caso de la empresa Cementos Progreso, con relación a una empresa cementera de la India (Valli, 2016), respecto al EVA como porcentaje del capital, que en ambos casos se sitúa alrededor de $8 \%$.

Respecto al índice Dupont, la comparación con empresas industriales de Perú (Arellanos, 2016) y de Colombia (Godoy, Acero y Rivera, 2011) muestra similitudes, en especial con la empresa $C B C$, respecto a margen sobre ventas. En ambos países dicho indicador es menor a 5 \%. En el caso de la empresa Cementos Progreso, la comparación con un estudio para Cemex (Valdés, Martínez y Beltrán, 2020) muestra un menor margen sobre ventas en esta empresa, de $18 \%$ contra $6 \%$, respectivamente, y similar nivel de apalancamiento y de rotación de activos, de alrededor de $45 \%$, en ambas empresas. 


\section{Conclusiones}

Las empresas pueden generar ganancias contables y rentabilidad sobre capital, pero no generar valor o hacerlo en monto insuficiente, como se evidenció con algunas empresas analizadas en este artículo.

De la muestra de diez empresas analizadas, se determinó que las cinco que emitieron bonos en el mercado internacional de deuda generaron mayor valor económico agregado que las cinco emisoras en el mercado nacional. Las primeras también evidenciaron mejor desempeño financiero según el método Dupont.

El análisis de desempeño financiero con los métodos EVA y Dupont es útil para que las empresas conozcan el monto y fuentes de creación de valor o su rentabilidad, en lo que respecta a un modelo de negocios basado en margen o volumen. 


\section{Referencias}

Alfaro, D., Castillo, J. y Gutiérrez, N. (2011). Análisis de la creación de valor económico para empresas del sector textil. TEC Empresarial, 1, (5), 41-48.

Arellanos, M. (2016). Evaluación del desempeño financiero del sector de empresas industriales peruanas, período 2010-2014. Revista Ex Cathedra en negocios, 1 (1), 18-28.

Bonilla, F. (2010). El valor económico agregado (EVA) en el valor del negocio. Revista Nacional de Administración, 1, (1), 55-70.

Desai, M. y Ferri, F. (2006). Understanding Economic Value Added. Harvard Business School.

Escobar, G. (2008). Las empresas de alimentos de Manizales generan ganancias pero destruyen valor económico agregado (EVA). Anfora, 15, (25), 317-336.

García, O. (2014). Fórmula Dupont y su rentabilidad, vista desde la óptica administrativa. Inquietud empresarial, XIV, (2), 89-113.

Gitman, L. y Joenhk, M. (2009). Fundamentos de inversiones. Pearson Educación.

Godoy, J., Acero, D. y Rivera, J. (2011). Análisis del desempeño financiero de empresas innovadoras del sector de alimentos y bebidas en Colombia. Pensamiento \& Gestión, 31, 109-136.

Granel, M. (2020). Rankia. https://www.rankia.cl/blog/analisis-ipsa/3926169sistema-dupont-para-analisis-rentabilidad

Pabón, J., Bastos, L. y Mongrovejo, J. (2015). Valor económico agregado en las empresas del sector industrial manufacturero de Cúcuta período 2008-2012. Respuestas, 2, (20), 54-72.

Quemada, E. (2016). FIT. Estrategia, valor y precio. Maximizar el verdadero valor de la empresa. Ediciones Pirámide.

Quichimbo, X. (2019). La rentabilidad ajustada al riesgo según el índice financiero (EVA) aplicado a cooperativas, 2016-2018. [Tesis de Maestría, Universidad del Azuay].

Téllez, J. (2015). Un análisis empírico de la creación de valor de las empresas que cotizan en la Bolsa Mexicana de Valores. [Tesis de Doctorado.] Universidad de Cantabria.

Valdés, F., Martínez, M., y Beltrán, J. (2020). Aplicación del método Dupont en el análisis de los motores de rentabilidad: Caso Cemex 2005-2019. Revista de Investigación Latinoamericana de Competitividad Organizacional, 2 (8), 15-29. 
Valli, A. (2016). A study on economic value added with special reference to the indian cements limited Tirunilvelli. International Journal of Innovative Research in Management Studies, 1, (4), 20-26.

Westreicher, G. (2020). Economipedia. https://economipedia.com/analisiseconomia/analisis-dupont.html 